\title{
О РАСШИРЕНИЯХ СИЛЬНО РЕГУЛЯРНЫХ ГРАФОВ БЕЗ ТРЕУГОЛЬНИКОВ С СОБСТВЕННЫМ ЗНАЧЕНИЕМ 4
}

\author{
И.Н. Белоусов, А.А. Махнев ${ }^{1}$, \\ ${ }^{1}$ Институт математики им. Н.Н. Красовского УрО РАН, Ковалевской 16, 620990 Екатеринбург, Россия \\ i_belousov@mail.ru, makhnev@imm.uran.ru
}

Мы рассматриваем неориентированные графы без петель и кратных ребер. Для вершины $a$ графа $\Gamma$ через $\Gamma_{i}(a)$ обозначим $i$-окрестность вершины $a$, то есть, подграф, индуцированный $\Gamma$ на множестве всех вершин, находящихся на расстоянии $i$ от $a$. Подграф $\Gamma(a)=\Gamma_{1}(a)$ называется окрестностью вершины $a$ и обозначается $[a]$, если граф $\Gamma$ фиксирован.

Степенью вершины называется число вершин в ее окрестности. Граф $Г$ называется регулярным степени $k$, если степень любой вершины $a$ из $\Gamma$ равна $k$. Граф $\Gamma$ назовем реберно регулярным с параметрами $(v, k, \lambda)$, если он содержит $v$ вершин, регулярен степени $k$, и каждое его ребро лежит в $\lambda$ треугольниках. Граф $\Gamma$ - вполне регулярный граф с параметрами $(v, k, \lambda, \mu)$, если он реберно регулярен с соответствующими параметрами, и $[a] \cap[b]$ содержит точно $\mu$ вершин для любых двух вершин $a, b$, находящихся на расстоянии 2 в $Г$. Вполне регулярный граф называется сильно регулярным графом, если он имеет диаметр 2. Графом в половинном случае называется сильно регулярный граф с параметрами $(4 \mu+1,2 \mu, \mu-1, \mu)$.

Если вершины $u, w$ находятся на расстоянии $i$ в $\Gamma$, то через $b_{i}(u, w)$ (через $c_{i}(u, w)$ ) обозначим число вершин в пересечении $\Gamma_{i+1}(u)\left(\Gamma_{i-1}(u)\right)$ с $[w]$. Граф $\Gamma$ диаметра $d$ называется дистанционно регулярным с массивом пересечений $\left\{b_{0}, b_{1}, \ldots, b_{d-1} ; c_{1}, \ldots, c_{d}\right\}$, если значения $b_{i}(u, w)$ и $c_{i}(u, w)$ не зависят от выбора вершин $u, w$ на расстоянии $i$ в $\Gamma$ для любого $i=0, \ldots, d$. Графом Тэйлора называется дистанционно регулярный граф с массивом пересечений $\{k, \mu, 1 ; 1, \mu, k\}$.

Дж. Кулен предложил задачу изучения дистанционно регулярных графов, в которых окрестности вершин - сильно регулярные графы со вторым собственным значением, не большим $t$, для данного натурального числа $t$. В настоящее время задача Кулена полностью решена для $t=3$.

В [1] начато решение задачи Кулена для $t=4$. А именно получена редекция к опрестностям вершин, являющимся исключительными графами. Нетрудно доказать, что сильно регулярный граф без треугольников с неглавным собственным значением 4 имеет параметры $(352,26,0,2),(352,36,0,4),(392,46,0,6),(552,76,0,12),(667,96,0,16)$ или $(784,116,0,20)$.

В данной статье изучены графы, в которых окрестности вершин имеют вышеуказанные параметры, причем $v \geq 392$. Ранее, в [2] было доказано, что дистанционно регулярный граф, в котором окрестности вершин - сильно регулярные графы с параметрами $(352,36,0,4)$, является сильно регулярным графом с параметрами $(9593,352,36,12)$. Случай окрестностей с параметрами $(352,26,0,2)$ является очень трудным (граница для диаметра имеет вид $d \leq$ 26).

Теорема. Граф, в котором окрестности вершин - сильно регулярные графы с параметрами $(392,46,0,6),(552,76,0,12),(667,96,0,16)$ или $(784,116,0,20)$ не является дистаницонно регулярным.

Работа выполнена при финансовой поддержке гранта РНФ (проект 14-11-00061).

\section{Литература}

1. Махнев А.А. Сильно регулярные графы с неглавным собственным значением 4 и их расширения // Известия Гомельского госуниверситета. 2014. Т. 84. № 3. С. 84-85.

2. Махнев А.А., Нирова М.С. О графах, в которых окрестности вершин - сильно регулярные графы с параметрами $(352,36,0,4)$ // Алгебра и приложения. Труды Межд. алгебр. конф. Нальчик. 2014 C. $84-85$. 